\title{
The Mystery on the Physical Conditions for Life
}

\author{
Kai Wai Wong1, Wan Ki Chow ${ }^{2}$ \\ ${ }^{1}$ Department of Physics and Astronomy, University of Kansas, Lawrence, USA \\ ${ }^{2}$ Department of Building Services Engineering, The Hong Kong Polytechnic University, Hong Kong, China \\ Email: kww88ng@gmail.com
}

How to cite this paper: Wong, K.W. and Chow, W.K. (2021) The Mystery on the Physical Conditions for Life. Open Journal of Biophysics, 11, 383-396.

https://doi.org/10.4236/ojbiphy.2021.114015

Received: August 3, 2021

Accepted: September 3, 2021

Published: September 6, 2021

Copyright $\odot 2021$ by author(s) and Scientific Research Publishing Inc. This work is licensed under the Creative Commons Attribution International License (CC BY 4.0).

http://creativecommons.org/licenses/by/4.0/ (c) (i) Open Access

\begin{abstract}
Based on the Perelmann mappings from the homogenous 5D onto the inhomogeneous Lorentz 4D manifold at liquid water phase temperature range, it is shown that coherent and decoherent molecules, water, carbon, hydrogen and oxygen naturally replace the Quarks in the standard model at Bethe fusion temperature as the $\mathrm{SU}(3)$ generators, leading to the formation of nitrogenous bases to house the monopole boson eigenstates that can exist within the RNA and DNA. Through which the growth of organic cells by inducing the Off Diagonal Long-Range Order of "p" type hole states in organic rings and bio-cells and thus "Life".
\end{abstract}

\section{Keywords}

Perelmann Mappings, RNA, DNA, Coherent and Decoherent Molecules Including Nitrogenous Bases, DNA Segments and Deceases, Intelligence, Senses and Memory

\section{Introduction}

We had previously proposed that the creation of the universe started with the creation of a homogeneous 5D manifold [1], and obeying the uncertainty principle. Such a manifold [2] then has an enclosing 4D homogenous Maxwell manifold boundary [3]. Because of that, the universe started with a Big Bang, with infinite energy, carried by the Diagonal Long-Range Order (DLRO) monopoles, and photons [4]. Since the monopoles are of DLRO, they must be in the Bose-Einstein ground state [5] [6]. It is this phenomenon that these monopoles are in fact the Higgs Bosons [7]. But because the existence of the simultaneous photons on creation requires the presence of a charge current, and as the monopole magnitude is composed of the direct products of e and -e opposite momenta pairs of massless e-trino, and anti-e-trino, the photons must arise from the natural breaking 
of the charge neutrality of the monopoles into equal numbers of massive e and -e Dirac spinors, that obey gauge invariance. It is this necessity so that charge currents can be generated to produce the photons during creation, that we can obtain via the expansion of the Bose distribution for the monopoles in terms of a converging power series of the Boltzmann partition factor, such that we can obtain the massive e and -e particle energies by truncating the lower limit of the Boltzmann factor $E / k T$, so that we can obtain different temperature depending on mass creations [8], such as the Gell-Mann standard model at Bethe fusion Temperatures, etc. [9], and including at low temperatures where the liquid water phase exists [10]. It is in this temperature range that we found the existence of Life forms on earth. As $T$ decreases so must the cut-off $E$ value. By applying the Perelmann's Ricci-flow entropy mapping, a toroidal Lorentz manifold is created, within time $t_{o}$, which then gives the toroidal tube radius $r_{o}=c t_{o}$ according to Fermat's sum, but with arbitrary toroidal center hole dimension; implying the Perelmann mapping can be initiated at an arbitrary time after the Big Bang. Hence $E_{o}$ in the lower cut-off obeying uncertainty is less than $h / t_{o}$. Should we accept Geesink's assignment that water represents the basic Cartan identity if the Lorentz space-time toroidal mapped out is completely uniformly occupied only by water molecules, then water's molecular weight of 18 nucleon mass must contain exactly the Cartan identity sum. Furthermore, the Geesink formula for coherent phonon spectra is completely valid.

The Quark model can be obtained by making a conformal projection of the 4 th space dimension into the remaining 3 space dimensions [1]. Since projection has no inverse, it is irreversible, so is making a Perelmann-Ricci-flow entropy mapping. Finally by exciting the Bose-Einstein ground state of the monopoles by expanding the Bose distribution in an increasing power series of the Boltzmann partition function and truncating the factor $E / k T$ at a lower limit cut-off for creating masses out of the monopole states is equivalent to the Higgs theory and is also irreversible. Thereby when we consider in the low temperatures, as life forms are created, these monopole states in the bio RNA and DNA systems must also be governed by irreversible geometrical boundaries; from the molecular structures that must contain not only coherent but also decoherent spectra that make up the spectra of the RNAs and DNAs. As life forms are only known to exist in such a low-temperature environment, it would be nice if we can deduce this fact from basic mathematical and physical principles.

\section{The Geesink Coherent Molecule}

It has always been the most mysterious question to us human as to how and under what necessary conditions can life forms exist on a planet. 75 years ago, Schrodinger the physicist that initiated Quantum Mechanics postulated that "LIFE" is also a quantum phenomenon [11]. Yet this connection remains unanswered. While it is well-known that water plays a necessary and key role. And to this day, we make it our goal to find water on other planets as our condition 
for hope of also finding life. More recently, Geesink and Meijer [12] successfully derived a low frequency spectra formula for bio-molecules, starting with water, as represented by a toroidal structure, such that other elements essential to life, such as oxygen, carbon, nitrogen and hydrogen can all be fitted into the same formula, dividing it into coherent and decoherent [13]. Because their formula fit so well to our knowledge of bio-rings, it is very interesting to investigate the Geesink coherent, decoherent spectra formulae which is based on the molecular weight, by standardizing the water molecular weight as 1 :

$$
E_{n, m, p}=h w_{r e f} 2^{n} 3^{m} 2^{p}
$$

where $w_{\text {ref }}$ is a cell specific frequency, assuming the Debye phonon spectrum dependence $M^{-0.5}$ on the molecular weight $M$, and $p, m$ are positive and negative integers. $n=0,0.5,2,4,5,7,8,-1,-3,-4,-6,-7$.

The 0.5 factor comes from hydrogen atomic weight which is $1 / 2 \times(1 / 3)^{2}$ as will become clearer as we discuss the essential molecules for life: water molecular weight as 1 representing the $\mathrm{SU}(3)$ generators Cartan identity, together with oxygen and carbon.

$$
\begin{gathered}
m=0,1,2,3,4,5,-1,-2,-3,-4,-5 . \\
p=<-4,-4,-3,-2,-1,0,1,2,3,4,5,6>52 .
\end{gathered}
$$

The weight of a molecule is overwhelmingly due to the constituents' atomic weight, as the electrons are only of order $1 / 1000$ of the nucleus. Thus, using the proton and neutron weight scale as 1 , water has a nucleon weight of $16+2=18$.

Geesink proposes that water should have a topological shape of a toroidal. According to the $\mathrm{SU}$ (3) Cartan identity, it would be in turns of $[2 / 3]^{2}+[2 / 3]^{2}+[-1 / 3]^{2}=1$. This Cartan Sum Rule then implies a toroidal Lorentz manifold as created out by Perelmann Ricci-Flow mapping, with the tube radius represented by $1 / 3$ as shown in Figure 1 below.

The tube radius $1 / 3$ is in unit of $r_{o}=c t_{o}$, where $t_{o}$ is the Ricci Flow action time.

The toroidal center core radius remains arbitrary, in agreement with Geesink's topological shape [14]. We like to point out that this is in agreement with Perelmann-Ricci-flow mapping [15], a necessary topological mapping that will create a 4D Lorentz manifold out of the 5D homogenous manifold.

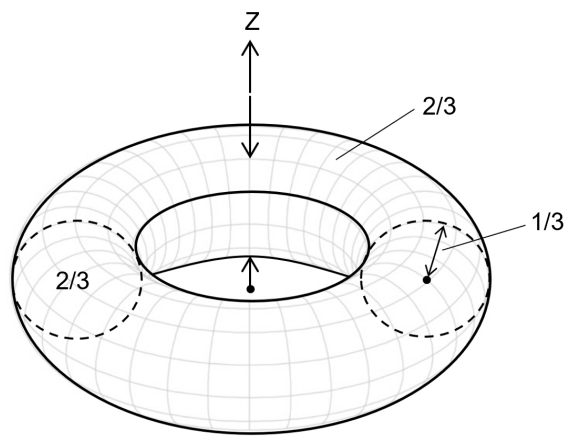

Figure 1. Geesink's toroidal model for water. 
Now considering the oxygen $\mathrm{O}_{2}$ molecule, we have: $[32 / 18]=1+7 / 9=1+[2 / 3]^{2}+3[1 / 3]^{2}$, which is a toroidal with attachments geometry.

Hydrogen $\mathrm{H}_{2}=2 / 18=[1 / 3]^{2}$ is only line geometry.

The combination water can be expressed as:

$$
\left[[16 / 18]+[1 / 3]^{2}\right]=\left[2(2 / 3)^{2}+(1 / 3)^{2}\right]
$$

which is indeed the simple $\mathrm{SU}(3)$ toroidal. That means it represents the realized Perelmann Ricci-flow mapping, $2 \mathrm{D} \times 1 \mathrm{D}$ toroidal space form. Thus the quadratic sum represents such $2 \mathrm{D} \times 1 \mathrm{D}$ symmetry, and is not given by the $3 \mathrm{D}$ Lorentz gauge. For that to be obeyed, we need the linear sum of the SU(3) generators.

To understand this 3D symmetry, let us investigate the Carbon 12 nucleus [16]. On it, we have 6 protons and 6 neutrons, arranged into three hexagons and 6 equilateral triangles. According to the Gell-Mann standard model, the proton and neutron are the gauge invariant product of three quarks: namely up-up-down and up-down-down. The up Quark has a fractional charge [2/3]e, while the down Quark is given by -[1/3]e. And 2/3, 2/3 and $-1 / 3$ form the set of SU(3) generators. Thus the gauge invariance restricts the linear sum of these generators to integer. Since carbon 12 nucleus is a spherical object, the gauge implied linear sum rule is applied to 3D space symmetry, and not that of the toroidal geometry [17]. Nonetheless, on the nuclear surface, it is still 2D, and the hexagon and triangles are and must be the mapping result similar to that suggested by Geesink for water. In fact, we can go a step further, and look at the surface of the Bucky Ball. There we found on top of hexagons, we have pentagons, replacing the triangles. It is this very feature in $2 \mathrm{D}$, which we find all the bio-rings are given in terms of hexagons, pentagons and triangles. There are no other geometrical shapes. Hence, the Geesink picture for water indeed should be valid.

Yet choosing water molecular weight as 1 is also equivalent to choosing it to represent the SU(2) Cartan generator 1, which makes the 5D to $4 \mathrm{D}$ mapping spherically symmetric. The Oxygen, and Nitrogen atom and molecule however always remain expressed as SU(3) generators only and like carbon are essential to the Lorentz 4D Ricci-flow toroidal mapping. In short for the coherent molecular absorption coherent spectra, water, oxygen, carbon and nitrogen plus hydrogen molecule play the role in determining the $\mathrm{SU}(3)$ generators in creation, while odd number of hydrogen atom leads to decoherent absorption, vital to the implement of evolution.

Now consider the molecule $2\left[\mathrm{CH}_{3}\right]$, its molecular weight is:

$$
30 / 18=\left\{1+(2 / 3)^{2}+2(1 / 3)^{2}\right\}
$$

Thus $\mathrm{CH}_{3}$ is an essential building block of bio-molecules similar to water "plus carbon".

$2\left(\mathrm{CH}_{3}\right)$ as represented in terms of the $\mathrm{SU}(3)$ generators is then clearly a coherence molecule. On the other hand, the single $\mathrm{CH}_{3}$ gives us in units of water $M=[1 / 2+1 / 3]$. The $1 / 2$ factor makes it a decoherent molecule. Thus separating 
of the two $\mathrm{CH}_{3}$ in a bio system will cost a breaking of symmetry.

It is this equivalence that allow yeast, a small bio-cell, to convert bio-carbonate powder, i.e., any baking flour, when water is added to create $\mathrm{CH}_{3}$, and thus gives to a physical rise in the mixture. The reverse is also true, when microwave is applied to baked bread releases water, and becomes moist. All bio-cells including yeast, contains a genome spectrum, thus nitrogenous bases.

Of the most important and interesting bio-rings are therefore the nitrogenous bases that make up the RNAs and DNAs in human.

Let's look at the nitrogenous base Uracil. See Figure 2 [1].

It contains $\mathrm{O}_{2}+2 \mathrm{H}_{2}+\mathrm{N}_{2}+4 \mathrm{C}$. Thus its molecular weight normalized to water is:

$$
\begin{aligned}
& \left\{1+(2 / 3)^{2}+3(1 / 3)^{2}\right\}+2(1 / 3)^{2}+\left\{1+(2 / 3)^{2}+(1 / 3)^{2}\right\}+4(2 / 3) \\
& =\left\{1+2(2 / 3)^{2}+6(1 / 3)^{2}+4(2 / 3)\right\}=\left\{2+5(1 / 3)^{2}+6(2 / 3)^{2}\right\} \\
& =\left\{3+4(1 / 3)^{2}+4(2 / 3)^{2}\right\}=\left\{5+2(1 / 3)^{2}\right\}
\end{aligned}
$$

The $2(1 / 3)^{2}$ denotes the two different dangling attachments of either $\mathrm{O}$ or $\mathrm{H}$ to the hexagon vertices. While the 5 denotes the topological degrees of freedom from 3 vertical axis choices +2 parities of the toroidal structure, represented by the hexagon.

And a clear indication of a toroidal of tube of radius (1/3), similar to water, and is actually resulted from the covariant Carbon in the nitrogenous base. The molecular weight of carbon $2 / 3$ can also be expressed as $(2 / 3)^{2}+2(1 / 3)^{2}$. Meaning it is both symmetric in structure to $3 \mathrm{D}$ and $2 \mathrm{D} \times 1 \mathrm{D}$. No other essential elements can do that. Thus due to this, carbon is vital to the formation of bio-rings.

Now let us investigate the corresponding end cap nitrogenous base in the DNA, namely Thymine.

It consists of the same hexagon ring of $4 \mathrm{C}$ and $2 \mathrm{~N}$, with the change in attachments of $2 \mathrm{O}, 2 \mathrm{H}$ and $\mathrm{H}_{3} \mathrm{C}$. Comparing to Uracil, the only difference is an extra $\mathrm{H}_{2} \mathrm{C}$ molecular weight.

$$
\mathrm{H}_{2} \mathrm{C}=14 / 18=7 / 9=(2 / 3)^{2}+3(1 / 3)^{2}
$$

Thus the weight becomes $\left\{5+2(2 / 3)^{2}+(1 / 3)^{2}\right\}=6$ exactly. This exact factor 6 for Uracil corresponds to the 3 vertical axis passing through the 3 adjacent Carbons in the hexagon times the 2 up, down faces differences instead of the 2 additive parities, making it a reflecting end cap on one face, and a transmitting through the reverse side. Such a property is then vital to allow for the monopole boson fields as discussed in our recent paper [14] to go around from one RNA side to loop around to the other RNA and thereby complete a quantum loop.

To further prove the point, let us consider another in between nitrogenous base Cytosine. It differs from Uracil, by missing an $\mathrm{H}$, and replacing a $\mathrm{O}$ with $\mathrm{NH}_{2}$. Thus we get the molecular weight as:

$$
\begin{aligned}
& \left\{5+2(1 / 3)^{2}\right\}-(1 / 3)^{2} / 2-2(2 / 3)^{2}+2(2 / 3)^{2} \\
& =\left\{5+(3 / 2)(1 / 3)^{2}\right\}=\left\{5+3(1 / 3)^{2}(1 / 2)\right\}
\end{aligned}
$$


<smiles></smiles><smiles>[H][R]n1nc2c(n1)C(=O)NC(NP)N2</smiles>

Adenine $\mathrm{A}$<smiles>N=c1nc(N)[nH]c2[nH]cnc12</smiles><smiles>CC(C)C1NC=CC(O)N(C)C1=O</smiles>

Nitrogenous
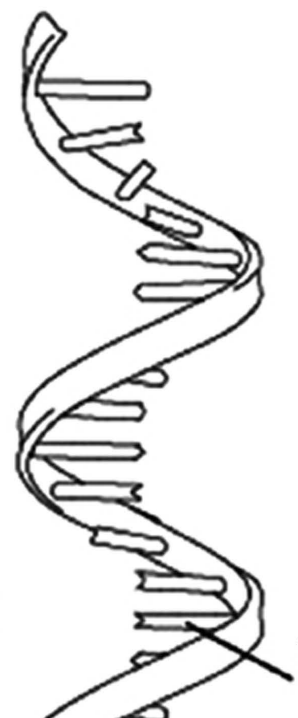

Nitrogenous Bases
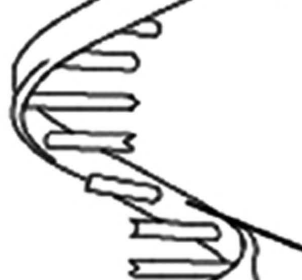

Bases pair

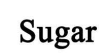
phosphate backbone

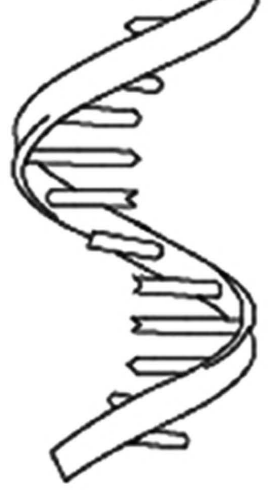

RNA

Ribonucleic acid

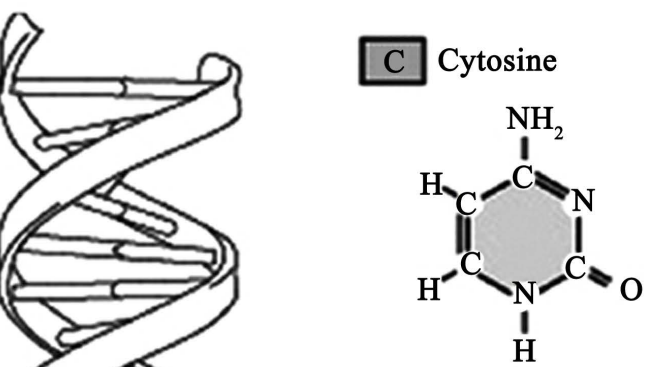

G Guanine<smiles></smiles>

A Adenine<smiles>[N]C1N=C(N)c2nc[nH]c2N1</smiles>

T Thymine<smiles>Cc1c[nH]c(=O)[nH]c1=O</smiles>

DNA

Dxeoxyribonueleic acid

\section{Nitrogenous Bases}

Figure 2. Images of DNA and RNA. Credit: NIH; adapted from national human genome research institute (Wong et al. 2014) [1].

The presence of the $(1 / 2)^{3}(1 / 3)^{2}$ term shows it is a decoherent ring structure, as the factor $1 / 2$ is not a Cartan generator. While the factor 5 is the degree of freedom 3 Cs and 2 parities.

Lastly let's consider the nitrogenous base Adenine. Compared again to cytosine, we replace $\mathrm{O}$ by $\mathrm{H}$, and add $\mathrm{CN}_{2}$. We get:

$$
\begin{aligned}
& \{5+(1 / 3)(1 / 2)\}-2(2 / 3)^{2}+(1 / 3)^{2}(1 / 2)+\left[(2 / 3)+\left\{1+(2 / 3)^{2}+(1 / 3)^{2}\right\}\right] \\
& =\left\{6+(1 / 2)(2 / 3)^{2}-(2 / 3)^{2}+(2 / 3)+(1 / 3)^{2}\right\} \\
& =\left\{6+3(1 / 3)^{2}+(2 / 3)^{2}(1 / 2)\right\}
\end{aligned}
$$


The factor 6 for the toroidal degrees of freedom of Adenine as compared to the factor 5 for Uracil is the result of the single pentagon attachment, making it having the extra degree of top and bottom, while the factor $3(1 / 3)^{2}$ is the result of parity with regard to the 3 perpendicular axis.

Again, Adenine contains a decoherent factor of $(2 / 3)^{2}(1 / 2)$ replacing the $3(1 / 3)^{2}(1 / 2)$ in Uracil.

In fact, the same decoherent factor exists for the remaining nitrogenous base Guanine. The only difference in molecular weight is an extra $\mathrm{O}$ and $\mathrm{H}$. Therefore, we have:

$$
\begin{aligned}
& \left\{6+3(1 / 3)^{2}+(2 / 3)^{2}(1 / 2)\right\}+17 / 18 \\
& =\left\{6+3(1 / 3)^{2}+\left[5(2 / 3)^{2}+(1 / 3)^{2}\right](1 / 2)\right\} \\
& =\left\{6+3(1 / 3)^{2}+\left[1+3(2 / 3)^{2}\right](1 / 2)\right\}
\end{aligned}
$$

It is vitally important to have the decoherent factor $(1 / 2)$, for the in-between nitrogenous bases so that the random stacking of all the bases can change and evolve giving rise to evolution and a distinct genome spectrum for each individual life. The decoherent part in Guanine contains a 1, representing the presence of a broken hexagon attachment to the important nitrogenous common carbon ring.

To be able to obtain these nitrogenous bases explicitly from Lie algebra mapping and its relationship to the different atoms will require much more topological mapping knowledge.

Nonetheless we see that Geesink's coherent and decoherent spectra formula indeed appears to be valid and plays a role in the understanding of the quantum aspect of Life as suggested by Schrodinger 75 years ago [18]. The phonon absorption spectra for these 5 nitrogenous bases were published by Geesink [14]. We present it in Figure 3 [14] simply for completeness.

The topological geometry of the nitrogenous bases does not change the quantum monopole loop spectra in the DNA, but it does provide a perturbation boundary condition effect to the DNA twisting itself, thus produces near infinite adjustment modifications possible, which in turn allows for the incoherent effect from the nitrogenous bases to induce the evolution of the life form, a very important physics that is built in for all survival of the fittest (See Chapter 12 of reference 1 , for which we will not repeat here).

\section{Survival of Life Forms}

To explain this survival of the fittest, let us consider the general structure of bio-cells. A similar creation of the bio-cells structures similar to the bio-rings geometry would result in point group like structures that also obey $2 \mathrm{D} \times 1 \mathrm{D}$ symmetry, which is similar to clay, which is composed of mainly silicon and the High Temperature Ceramic Superconductors [19]. These structures lead to semi-conductor like band structures, with positive band gap between the 


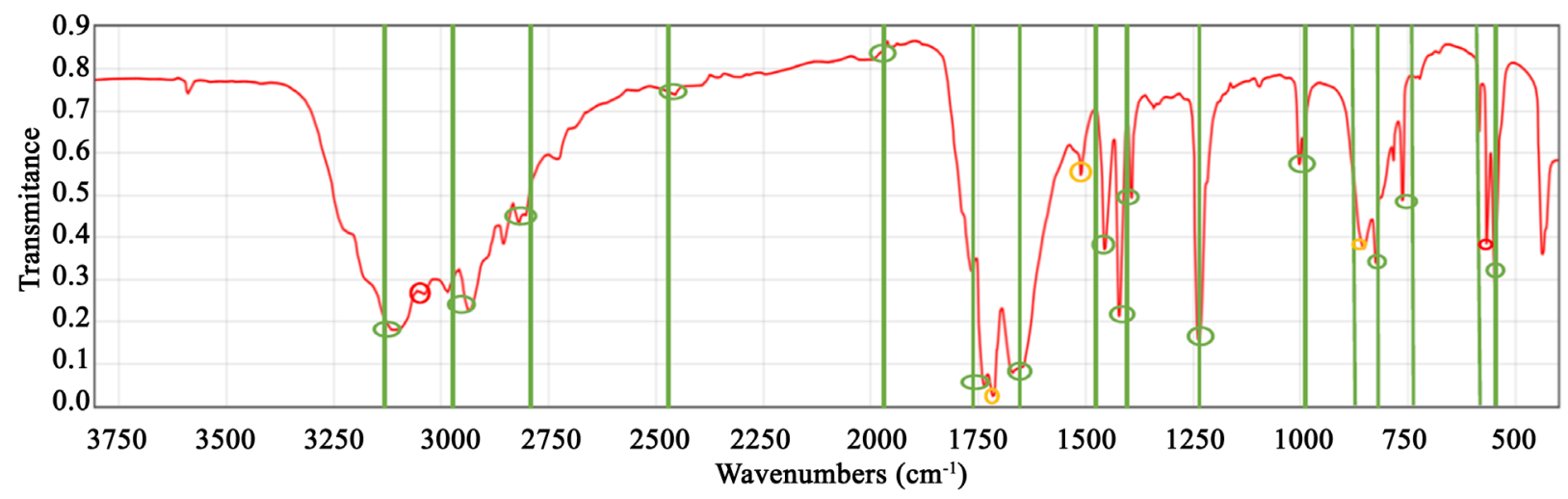

(a)

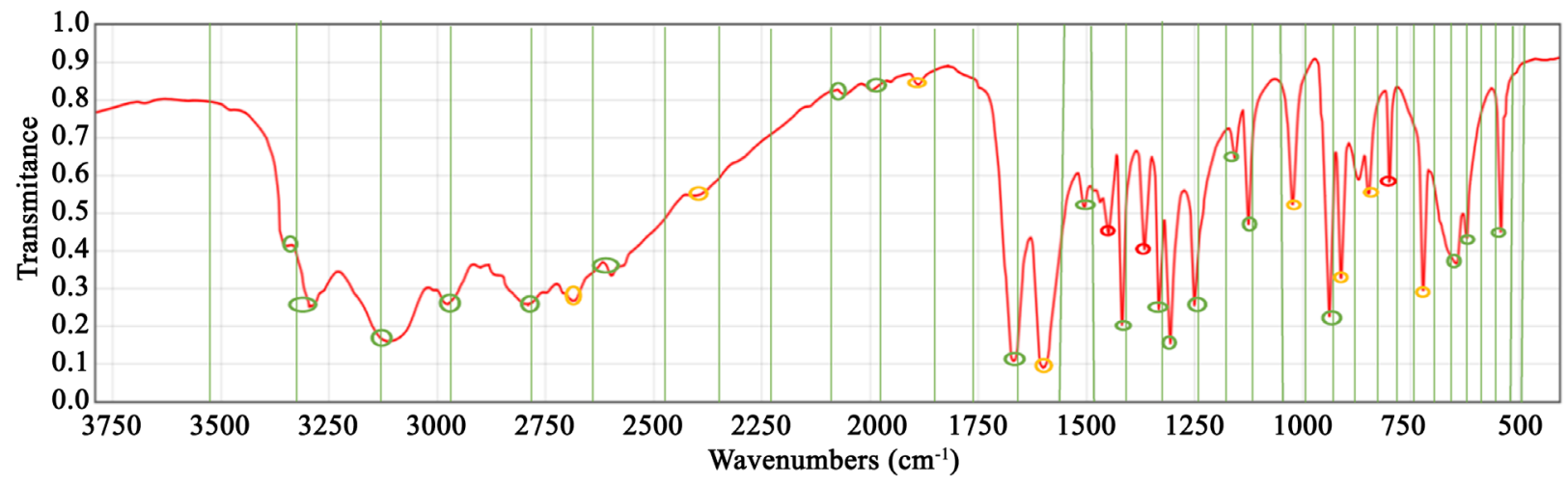

(b)

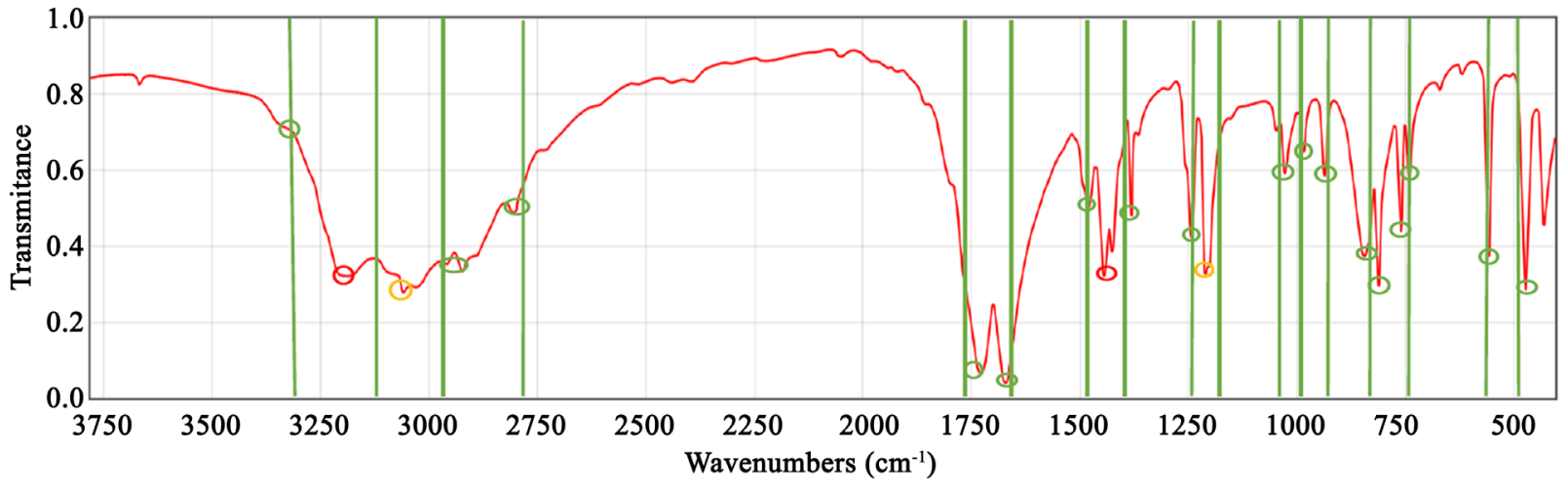

(c)

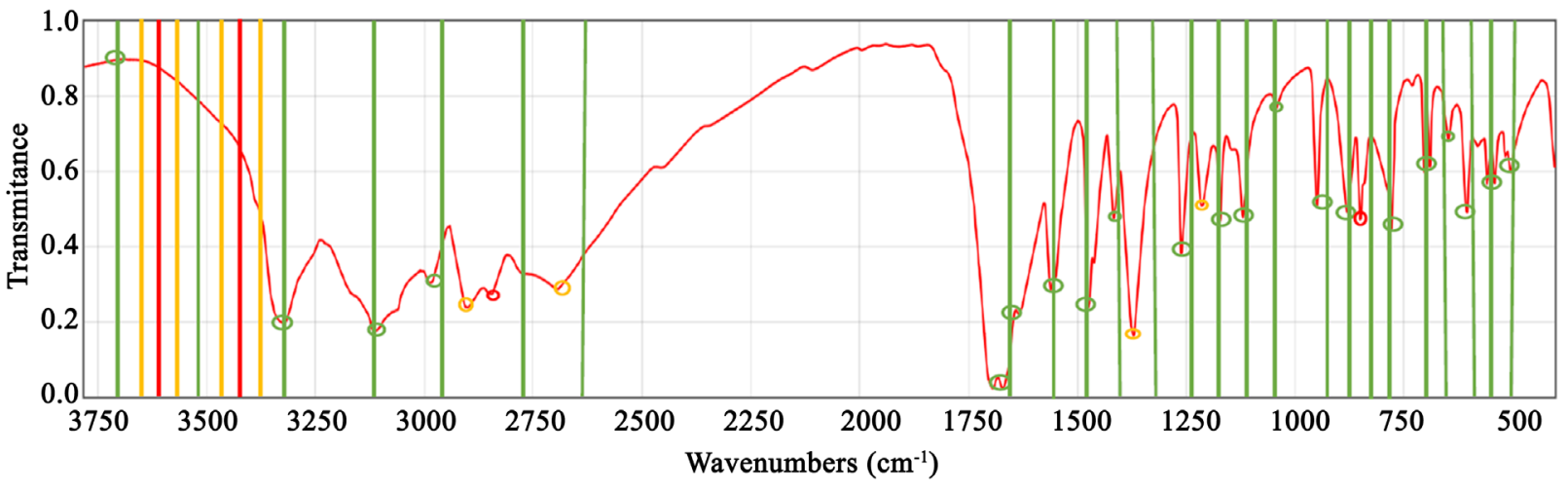

(d) 


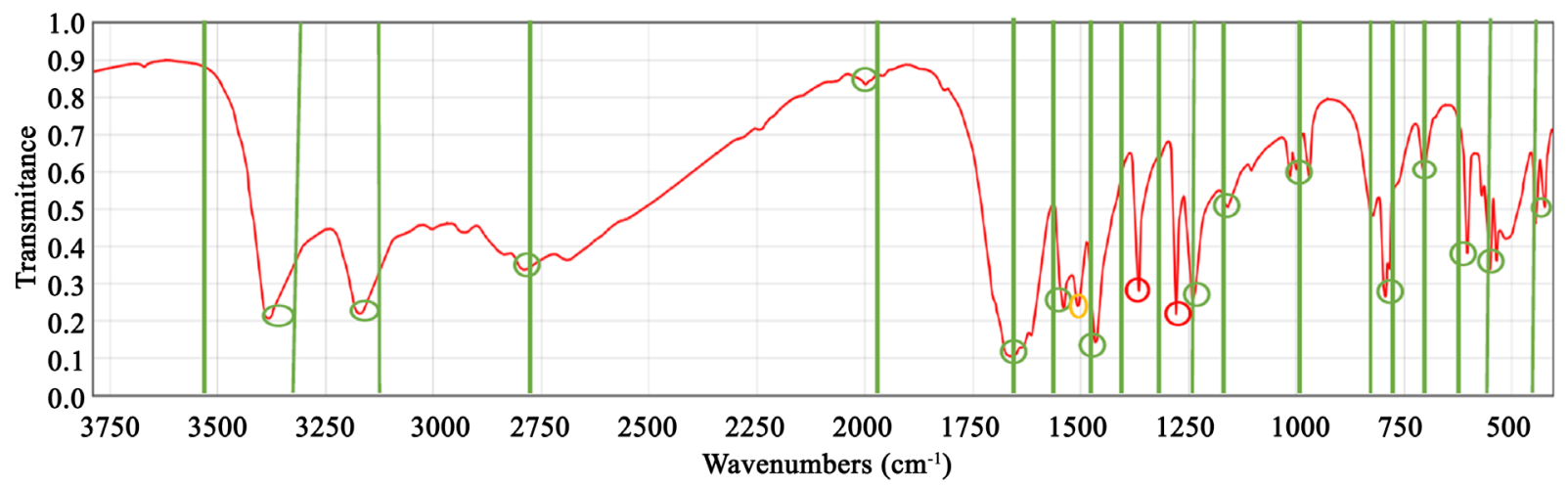

(e)

Figure 3. Phonon absorption spectra for the 5 nitrogenous bases (Geesink 2020) [14]. (a) Uracil, NIST. Notice: spectra from this collection were measured on dispersive instruments, often in carefully selected solvents, and hence may differ in detail from measurements on FTIR instruments or in other chemical environments. Remark: Overall pattern is predominantly coherent according to the proposed coherence-scale (green), partly in transition zone (yellow), and some frequencies (red) at decoherent positions; (b) Adenine, NIST. Remark: Overall pattern is predominantly coherent according to the proposed coherence-scale (green), partly in transition zone (yellow), and some frequencies (red) at decoherent positions; (c) Thymine, NIST. Remark: Overall pattern is predominantly coherent according to the proposed coherence-scale (green), partly in transition zone (yellow), and some frequencies (red) at decoherent positions; (d) Guanine, NIST. Remark: Overall pattern is predominantly coherent according to the proposed coherence-scale (green), partly in transition zone (yellow), and some frequencies (red) at decoherent positions; (e) Cytosine, NIST. Remark: Overall pattern is predominantly coherent according to the proposed coherence-scale (green), partly in transition zone (yellow), and some frequencies (red) at decoherent positions.

Valence Band (VB), and the empty Conduction Band (CB). Except that for the high temperature superconductors (HTC), the VB is partially filled, due to Oxygen in the $2 \mathrm{D}$ ring layer structure, quite like the $\mathrm{CuO}$ plane in HTC ceramics [20] (Figure 4, YBCO). Thus these bio-cells are $\mathrm{p}$ type conductors in its normal phase, and would become superconducting at Temperature $T$ below its superconducting transition $T c$, yet still within the water phase temperature range [21]. As that happens, the hole states become Bosons with Off Diagonal Long Range Order (ODLRO), with specific binding gap matching to extend the DLRO of the specific eigen-energy monopole bosons within the DNA of the Life form. This induced Long Range symmetry then provides the growth mechanism to the multiplying of the different bio-cells. The genome spectra in the DNA, thereby induce the multiplicity of the different bio-cells in the body. Similarly, the decoherent nitrogenous bases within the DNA causing the genome evolution lead to the death and rebirth with evolution of the bio-cells within the life form. Such evolution is expected to occur in all lives, from viruses to human from generation to the next, hence driving the reproduction of the life form under the survival of the fittest principle. Because of this processes, cancerous growth can also occur. Such cancerous growth can be separated into 2 sources. One that is usually inherited originates from the DNA, while the other caused by the bio-ring and proteins components in the bio-cells structure. Knowledge of these effects can allow for suitable cure approaches.

To investigate this potential cancerous problem, let us start from the formation of RNA and DNA. Note the RNA is a linear chain obtained from the stacking 


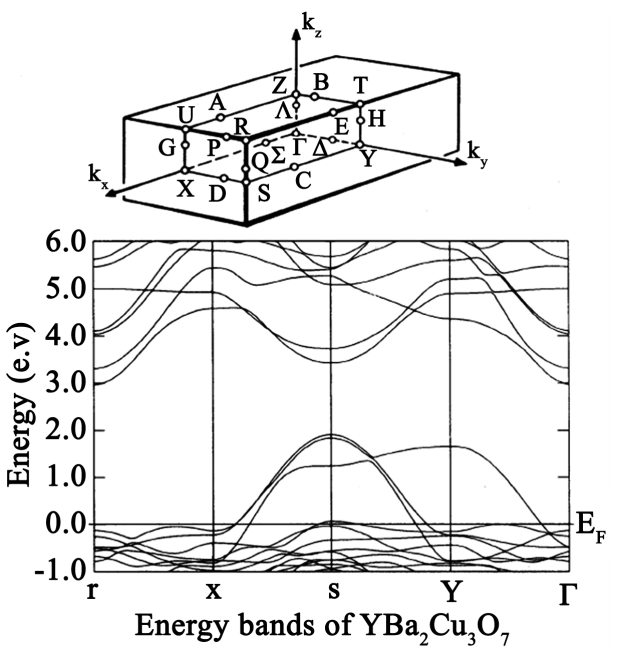

(a)

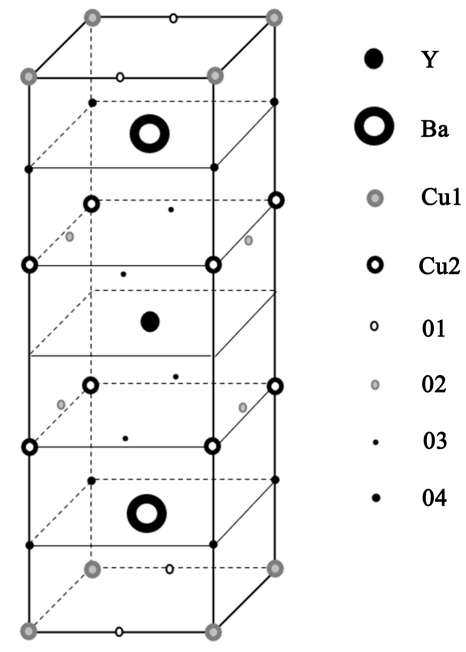

(b)

Figure 4. (a) Energy bands of $\mathrm{YBa}_{2} \mathrm{Cu}_{3} \mathrm{O}_{7}$ in the vicinity of the Fermi energy, and the "semiconductor" gap. Inset: The orthorhombic BZ (Wong and Ching, 2004) [20]. (b) The crystal unit cell structure of ceramic superconductor $\mathrm{YBa}_{2} \mathrm{Cu}_{3} \mathrm{O}_{7}$ (Ching et al. 1987) [19].

of the 4 nitrogenous bases, linked together due to a backbone built from the chain of Carbon from the N-C-N half of the hexagon of each nitrogenous base. There do not exist an Eigen-monopole state through this backbone, because the DLRO monopole Boson must satisfy the $\pi$ phase as mentioned in our previous paper [4]. The monopole state can exist by quantum tunneling from one nitrogenous base to the next from the remainder $\mathrm{C}-\mathrm{C}-\mathrm{C}$ in the hexagon. This monopole state however must also obey the rigid end boundary condition of the RNA. Thus it means of the 4 nitrogenous bases, one must act as an end cap. Or more preciously such an end cap base must violate parity symmetry, such that the monopole Boson can only tunnel through one face and not the reverse face. Hence the RNA can only be formed by the random stacking of 7 faces of the 4 nitrogenous bases. This monopole Eigen state then must satisfy the rigid boundary plus the phase matching as it quantum tunnels from each layer to the next adjacent layer. Obviously if the RNA linear chain is twisted due to the backbone twisting, then the phase matching condition is varied and the resulting eigenvalue choice can be infinite.

\section{The DNA and RNA Spectra}

As mentioned before, there are 7 independent faces from the 4 nitrogenous bases of both the RNA, and DNA from which the monopole DLRO bosons can exist linking the carbon cores within the nitrogenous 3 side by side carbon hexagon, as the backbone spirals, because one nitrogenous base must serve as the end cap. For the RNA, this end cap serves to be the fixed boundary for the boson, while for the DNA, it serves as a reflector such that the boson wave then goes from one RNA side to the other side, making each boson wave completes a close loop. There is no requirement that the intermediate stacking of the 7 faces of the ni- 
trogenous bases cannot be duplicated, nor can anyone be totally missing. Hence, there are an infinite variety of possible RNAs and DNAs. Only by imposing a no repeat condition can we limit the possibility. In fact, by such an imposition we can get a maximum genome number $N$ of $7 ! \times 3 \times 2=30240$. The factor 3 comes from the 3 side by side carbons tunneling, and the factor 2 comes from the $2 \mathrm{pa}$ rallel RNAs that make up the DNA. This number is in fact close to that reported for the human genome. That of course does not mean the non-repeat and exact number to be rigorously true for every human. Because each layer of nitrogenous base within the RNA must contain a separation space gap, the two RNAs that make up the DNA, then must be shifted so that they can fit into a tight line. Hence, it also implies these two RNAs can be separated, transforming the DNA structure into a closed ring instead of a line. To get the DNA into a line, it requires the DNA be twisted, by a pair of in-phase spiraling backbones, as shown in Figure 2. This 180 degree twisting, then divided the separated nitrogenous bases layers into equal segments of n layers each. Thus resulting in $S=N / n$ segments. Each of these segment Eigen-values of the DLRO bosons performs a special bodily function to inducing the ODLRO of the body cells/organs " $\mathrm{p}$ " holes formation, and thus producing the regenerating growth of it.

An error in the nitrogenous bases stacking will result in cancerous growth, and is likely to pass from one generation to the next, the so-called inherited diseases. Recently the biotech firm Intellia Therapeutics announced it is able to edit genes inside the body, based on CRISPR, originally discovered by Jennifer Doudna [22] a 2020 Nobel Prize in Chemistry winner. The method is to zero in the specific segment in the DNA, where the missed nitrogenous base occur that led to the specific organ problem, and just separate that specific segment into a doughnut-like ring, such that the correct nitrogenous base can be replaced and properly installed into the DNA. Intellia studied the specific disease "transthyretin" associated with the liver function, and by just replacing a few DNAs in the liver was shown successful. Of course, not all inherited diseases are as simple as transthyretin, and for how long the disease can be suppressed. Furthermore, direct nitrogenous base replacement can alter the DNA spectra unintentionally leading to unwanted side effects. In fact, the Intellia success in pinpointing the DNA segment where problem lies actually allows for a safer RNA interference (RNAi) approach, letting the body generate the proper DNA itself, such as the methods employed by Alnylam Pharmaceutical [23] and Rocket Pharmaceutical [24].

\section{Sensing and Intelligence}

The monopole boson states within RNA and DNA that govern the growth and reproduction of bio-cells do not provide animal life forms of its senses, intelligence and accumulation of knowledge. To realize that it is important to recognize that the DLRO monopole bosons require the quantum tunneling ability from within the carbon nuclear void core to the adjacent core. But most chemical elements do not possess a void core similar to carbon 12; for example silicon. Yet 
such elements, such as clay are equally created from the Perelmann Ricci-flow entropy mappings, and its molecular weight also obey the Geesink-Jerman-Meijer coherent and decoherent formula [25]. Its conducting electronic states can only be either as free electrons, such as in metals or else as insulators and semi-conductors. Only for metals in the bio-cell structures can it in principle be induced to form an ODLRO superconducting state. It is however well-known through the Bardeen-Cooper-Schrieffer (BCS) [26] theory due to the absence of the exciton mechanism [27], that metallic $T c$ value is far lower than room temperature, and hence fell out of the DLRO spectra range of the DNAs. In short, there are no electron carriers inducible to create bio-cells. The only activation by the monopole spectra energies is to excite the electron from the filled valence band into the empty conduction band of a coherent semi-conductor bio-molecule, by inducing an electric potential thus making the creation of a computer-like chip circuit possible. These chips circuit existence within an animal allows the animal to build senses, that is sight, sound, smell, taste and pain, plus intelligence as well as memory. Normally this brain center is concentrated in an enclosed bone structure called the head. The charge circuitry feeding the brain is of course still from bio-cells, such as bone marrow, and nerves, which could be cut by blockage at the so-called acupuncture points or become cancerous when we have missed nitrogenous bases within the DNA segments. As well as when the connecting nerve cells die they leave behind the accumulation of dead cells around the brain leading to signal blockage and thereby memory lost, bringing on Alzheimer [28].

\section{Conclusion}

We have briefly gone through how Life can be created through the topological Perelmann mappings of the homogenous 5D into the non-homogenous Lorentz $4 \mathrm{D}$ manifold at room temperature, that is the temperature range of liquid water. And through which coherent and decoherent molecules spectra, including the life-essential elements, water, oxygen and carbon, and clays that then allows for the forming of nitrogenous bases, building up RNA and DNA, so that DLRO monopole bosons spectra can induce the ODLRO of "p" type conducting hole states to create grow of cells and organs, while the clay structures are semi-conductors, and can be organized into computer-like chips, feed by conducting nerves, thus produces senses, intelligence and memory. Each segment of the DNA appears to control the physical function properties of a specific body organ. There are many and complex quantum chemistry, biology and medicine researches already accomplished and many more remain to be found. This over-simplified paper is just an attempt to put everything under a unified mathematical foundation that can be better understood? Hopefully, it can lead to scientifically sound across and combined usage of different known and new successful techniques in the treatment of both yet open inherited and acquired diseases, such as Alzheimer's.

\section{Acknowledgements}

We thank Ms. Winnie So for her tremendous help in the preparation of this 
manuscript.

\section{Conflicts of Interest}

The authors declare no conflicts of interest regarding the publication of this paper.

\section{References}

[1] Wong, K.W., Dreschhoff, G. and Jungner, H. (2014) The Five Dimension Universe: A Creation and Grand Unified Field Theory Model. Scientific Research Publication, Irvine.

[2] Aczel, A.D. (1997) Fermat's Last Theorem: Unlocking the Secret of an Ancient Mathematical Problem. Penguin Press, London.

[3] Maxwell, J.D. (1865) A Dynamic Theory of Electromagnetic Field. Philosophical Transactions of the Royal Society of London, 155, 459-512. https://doi.org/10.1098/rstl.1865.0008

[4] Wong, K.W., Dreschhoff, G., Jungner, H., Fung, P.C.W. and Chow, W.K. (2018) The Magnetic Monopole in 5D Homogeneous Space-Time. Physics Essays, 31, 493-495. https://doi.org/10.4006/0836-1398-31.4.493

[5] Wong, K.W. and Chow, W.K. (2021) A Summary of the Homogeneous 5D Universe Creation Model. Expressed in the Dirac Second-Order Quantization Representation. Journal of Modern Physics, 12, 123-138.

https://doi.org/10.4236/jmp.2021.123012

[6] Chow, W.K., Fung, P.C.W. and Wong, K.W. (1985) Bose-Einstein Condensation in Superfluid Helium Four. Journal of the Physical Society of Japan, 54, 4490-4493. https://doi.org/10.1143/JPSJ.54.4490

[7] Higgs, P.W. (1964) Broken Symmetries and the Masses of Gauge Bosons. Physical Review Letters, 13, 508-509. https://doi.org/10.1103/PhysRevLett.13.508

[8] Wong, K.W., Dreschhoff, G.A.M. and Jungner, H.J.N. (2012) The Homogeneous 5D Projection and Realization of Quark and Hadron Masses. arXiv: 1202.5761v1.

[9] Wong, K.W., Dreschhoff, G. and Jungner, H. (2012) On Neutrino Oscillation and Predicting the $125 \mathrm{GeV}$ Two Photon Emission State from p-p Collision Based on the 5D Homogeneous Space-Time Projection Model. Journal of Modern Physics, 3 , 1450-1457. http://dx.doi.org/10.4236/jmp.2012.310179

[10] Wong, K.W., Fung, P. and Chow, W.K. (2019) 5D Model Theory for Creating of Life Forms. Journal of Modern Physics, 10, 1548-1565.

https://doi.org/10.4236/jmp.2019.1013103

[11] Schrodinger, E. (1992) What Is Life? Cambridge University Press, New York. https://doi.org/10.1017/CBO9781139644129

[12] Geesink, H.J. and Meijer, D.K.F. (2018) A Semi-Harmonic Frequency Pattern: Organizes Local and Non-Local States by Quantum Entanglement in both EPR-Studies and Life Systems. Journal of Modern Physics, 9, 898-924. https://doi.org/10.4236/jmp.2018.95056

[13] Meijer, D.K.F., Jerman, I., Melkikh A.V. and Sbitrev V.I. (2020) Biophysics of Consciousness: A Scale-Invariant Acoustic Information Code of a Superfluid Quantum Space Guides the Mental Attribute of the Universe. In: Bandyopadhyay, A. and Ray, K., Eds., Rhythmic Oscillations in Proteins to Human Cognition Book, Springer/Nature Publisher, Singapore, 213-361.

[14] Geesink, H.J. (2020) Proposed Informational Code of Bio Molecules and Its Build- 
ing Blocks: Quantum Coherence versus Decoherence. Private Communication, preprint.

[15] Perelmann, G. (2002) The Entropy Formula for Ricci Flow and its Geometric Applications. ArXiv.math.DG/0211159.

[16] Wong, K.W. (1964) Application of Nonlocal Field Operators to a System of Hard Sphere Bose Gas. Journal of Mathematical Physics, 5, 637-642. https://doi.org/10.1063/1.1704157

[17] Perelmann, G. (2003) Ricci Flow with Surgery on Three-Manifolds. ArXiv.math.DG0303109.

[18] Frohlich, H., (1968) Long-Range Coherence and Energy Storage in Biological Systems. International Journal of Quantum Chemistry, 2, 641-649. https://doi.org/10.1002/qua.560020505

[19] Wong, K.W. and Ching, W.Y. (2004) A Structural-Based Microscopic Theory on High-Temperature Cuprate Superconductors. Physica C: Superconductivity, 416, 47-67. https://doi.org/10.1016/j.physc.2004.09.003

[20] Ching, W.Y., Xu, Y., Zhao, G.-L., Wong, K.W. and Zandiehnadem, F. (1987) Electronic Structure and Excitonic-Enhanced Superconducting Mechanism in $\mathrm{YBa}_{2} \mathrm{Cu}_{3} \mathrm{O}_{7-\delta}$, Physical Review Letters, 59, 1333-1336. https://doi.org/10.1103/PhysRevLett.59.1333

[21] Wong, K.W. and Curatolo, S. (2008) EEM: The Exciton Enhancement Mechanism Theory and Experimental Evidence of Optically Enhanced Tc in High Tc Superconductors. In: Chang, O.A., Ed., Progress in Superconductivity Research, Nova Science Publishers, Inc., Hauppauge, 55-78.

[22] CNBC (Consumer News and Business Channel) (2021) Intellia Therapeutic CEO John Leonard on Crispr Gene-Editing July 2nd, 2021.

[23] Yahoo Finance (2021, June 30) Alnylam Initiates KARDIA-1 Phase 2 Study of Zilebesiran (ALN-AGT) in Patients with Mild-to-Moderate Hypertension. https://finance.yahoo.com/news/alnylam-initiates-kardia-1-phase-110000305.html

[24] Yahoo Finance (2021, May 13) Rocket Pharmaceuticals Announces Positive Data from RP-L102, RP-L201 Gene Therapy Programs.

https://finance.yahoo.com/news/rocket-pharmaceuticals-announces-positive-data-1 60047724.html

[25] Geesink, H.J., Jerman, I. and Meijer, D.K.F. (2020) Clay Minerals Information Networking Quantum Coherence Versus Decoherence and First Life. Private Communication.

[26] Bardeen, J., Cooper, L.N. and Schrieffer, J.R. (1957) Theory of Metallic Superconductivity. Physical Review, 108, 1175-1204. https://doi.org/10.1103/PhysRev.108.1175

[27] Wong, K.W., Fung, P.C.W., Yeung, H.Y. and Kwok, W.Y. (1992) Analysis of Isotope Effect of High $T_{\mathrm{c}}$ Ceramic $\mathrm{YBa}_{2-\mathrm{x}} \mathrm{La}_{\mathrm{x}} \mathrm{Cu}_{3} \mathrm{O}_{7}$ Using the Excitonic Enhancement Model. Physical Review B, 45, 13017-13024. https://doi.org/10.1103/PhysRevB.45.13017

[28] ABC News (2021, 7 June) New Alzheimer's Drug Is 1st of Its Kind to Be FDA Approved.

https://abcnews.go.com/Health/alzheimers-drug-1st-kind-fda-approved/story?id=7 8128617 